\title{
Protocols and risks: when less is more
}

\author{
Jaspreet Pannu ${ }^{1 凶}$ Jonas B. Sandbrink ${ }^{2}$, Matthew Watson ${ }^{3}$, Megan J. Palmer ${ }^{4,5}$ and \\ David A. Relman $\mathbb{1}^{1,5,6,7 凶}$
}

ARISING From Xie, X. et al. Nat. Protoc. https://doi.org/10.1038/s41596-021-00491-8 (2021).

Xie et al. recently described 'a reverse genetic system that enables rapid synthesis of wild-type, mutant and reporter SARS-CoV-2 [severe acute respiratory syndrome coronavirus 2] strains' ${ }^{1}$. Their goal was to enable researchers to assess the functional properties of sequence variants, including their susceptibility to countermeasures such as vaccine-induced immune responses. We agree that the rapid development of medical countermeasures is of utmost importance, especially during an ongoing pandemic. However, we believe that public dissemination of this protocol in its current form poses risks that outweigh the benefits. In providing detailed, step-by-step instructions, it enables anyone skilled in the art anywhere in the world to create novel variants of SARS-CoV-2 more quickly, including variants that have even more worrisome properties than those that have occurred naturally. Sometimes research should be slowed, not hastened, to ensure a proper discussion of goals and a full and public vetting of proposed plans. Detailed protocols pose special risks under certain circumstances like this one and should undergo special prepublication scrutiny.

Reverse genetic systems have clearly facilitated the study of virus-host interactions and the development of antivirals and vaccines. Owing to the large size of coronavirus genomes, the instability of specific coronavirus cDNA sequences, and their propensity for acquiring mutations in bacteria, the development of these systems for coronaviruses has been especially challenging. The goal of the detailed protocol presented by Xie et al. is to enable 'rapid synthesis of wild-type, mutant and reporter SARS-CoV-2 strains' by using optimized cDNA clones that span the genome and an efficient electroporation procedure. The protocol describes many of the problems that currently plague practitioners; provides instructions for anticipating, avoiding and troubleshooting these problems; and offers a detailed 'shopping list' for needed reagents. In doing so, the authors increase the likelihood of, and decrease the time needed for, successful virus expression by coronavirus investigators. However, most dedicated investigators who need this capability have already acquired it to a large degree. At the same time, this protocol also lowers the barrier to entry for those with no direct experience or tacit knowledge or particular need, including for malicious actors. As the authors state, 'this protocol will enable researchers from different research backgrounds to master the use of the reverse genetic system'.

This protocol raises some important and difficult questions. How many people, and in what physical settings, do we want or need designing and producing novel coronaviruses? Should anyone be encouraged to synthesize SARS-CoV-2 recombinant viruses of their own design and choosing? What about a recombinant deliberately designed to be resistant to all known antibodies and cellular immune responses? At what point, especially given the high transmissibility of this virus, do the risks of misadventure, ill-conceived plans, poor oversight and inadequate laboratory safety override the benefits? By significantly increasing the number of practitioners, the authors directly increase the likelihood of, and decrease the time to, an accident, especially among inexperienced researchers without adequate biocontainment ${ }^{2}$.

Reverse genetic engineering enables modification of critical properties of pathogens such as transmissibility, virulence and immune evasion. The dissemination of this capability materially changes the risk landscape. Infectious agents with high degrees of transmissibility pose special risks per se because our ability to manage these agents once they have escaped containment is limited at best. SARS-CoV-2 has made this point very clear. Although similar reverse genetic protocols have indeed been published for SARS-CoV-1 and Middle East respiratory syndrome coronavirus (MERS-CoV), SARS-CoV-2 is more transmissible than either of these two viruses ${ }^{3}$. Since this protocol's publication, Amarilla et al. have developed a similar reverse genetics system, which they intend to be easily translatable to other positive-strand RNA viruses ${ }^{4}$. Although Amarilla et al. state that infectious full-length cDNA is readily assembled ... without the need for technically demanding intermediate steps $^{4}$, it is worth noting that their methods section is far less detailed than that of Xie et al. Efforts to develop protocols that empower many more actors with diverse motivations to

${ }^{1}$ Department of Medicine, Stanford University School of Medicine, Stanford, CA, USA. ${ }^{2}$ Medical Sciences Division, University of Oxford, Oxford, UK. ${ }^{3}$ Center for Health Security, Johns Hopkins Bloomberg School of Public Health, Baltimore, MD, USA. ${ }^{4}$ Department of Bioengineering, Stanford University, Stanford, CA, USA. ${ }^{5}$ Center for International Security and Cooperation, Freeman Spogli Institute for International Studies, Stanford University, Stanford, CA, USA. ${ }^{6}$ Department of Microbiology \& Immunology, Stanford University School of Medicine, Stanford, CA, USA. ${ }^{7}$ Infectious Diseases Section, Veterans Affairs Health Care System, Palo Alto, CA, USA. ${ }^{凶}$ e-mail: pannu@stanford.edu; relman@stanford.edu 
practice reverse genetic engineering of potentially dangerous infectious agents demand special scrutiny and review.

Concerns during the past decade about research on potential pandemic pathogens led the US Government to develop new guidance for the review of such research ${ }^{5}$. We understand that work by Xie et al. in support of their protocol was subject to local institutional review, given some of the concerns. However, in our view, this guidance, the resulting federal review process and most local reviews of work of this type lack sufficient clarity, scope, authority and transparency.

Previous dual-use controversy ${ }^{6,7}$ has resulted in proposals for risk mitigation that include limits on the amount and kinds of detail about the methods used in particularly risky research. For example, members of the National Science Advisory Board for Biosecurity have recommended that when work reveals a means for enhancing the transmissibility or pathogenicity of potential pandemic pathogens, 'the basic result be communicated without methods or details, [so] that the benefits to society are maximized and the risks minimized's

The publication of a how-to manual for work of this sort would seem to work in opposition to this risk-mitigation approach. Further guidance is needed for this genre of scientific publication. In the spirit of the mission of this journal, we need easy-to-use review protocols for assessing the safety and security of scientific protocols under conditions of pressing public health need. Risk mitigation might involve routine oversight via screening ${ }^{9}$ or registration for access to commercial synthesis of large, specific DNA constructs, such as the seven SARS-CoV-2 cDNA plasmid clones described by Xie et al. ${ }^{1}$. Other forms of risk mitigation might include public notification of intention to synthesize known or potential pandemic pathogens, or public disclosure of intended, new modifications to a known pandemic agent. There are circumstances when faster is not better, or even smart. Adequate review at a minimum requires established frameworks and processes for assessing risk throughout the research lifecycle and the involvement of experts with diverse perspectives ${ }^{10,11}$.

\section{References}

1. Xie, X. et al. Engineering SARS-CoV-2 using a reverse genetic system. Nat. Protoc. 16, 1761-1784 (2021).

2. Perrow, C. Normal Accidents: Living with High Risk Technologies updated edn (Princeton University Press, 2011).

3. Petersen, E. et al. Comparing SARS-CoV-2 with SARS-CoV and influenza pandemics. Lancet Infect. Dis. 20, e238-e244 (2020).
4. Amarilla, A. A. et al. A versatile reverse genetics platform for SARS-CoV-2 and other positive-strand RNA viruses. Nat. Commun. 12, 3431 (2021).

5. US Department of Health and Human Services. Framework for Guiding Funding Decisions about Proposed Research Involving Enhanced Potential Pandemic Pathogens. https://www.phe.gov/s3/ dualuse/documents/p3co.pdf (2017).

6. Herfst, S. et al. Airborne transmission of influenza A/H5N1 virus between ferrets. Science 336, 1534-1541 (2012).

7. Noyce, R. S., Lederman, S. \& Evans, D. H. Construction of an infectious horsepox virus vaccine from chemically synthesized DNA fragments. PloS One 13, e0188453 (2018).

8. Berns, K. I. et al. Adaptations of avian flu virus are a cause for concern. Science 335, 660-661 (2012).

9. World Economic Forum. Biosecurity Innovation and Risk Reduction: A Global Framework for Accessible, Safe and Secure DNA Synthesis (in collaboration with the Nuclear Threat Initiative). https://www.weforum.org/reports/biosecurity-innovation-and-riskreduction-a-global-framework-for-accessible-safe-and-secure-dnasynthesis-582d582cd4/ (2020).

10. Evans, S. W. et al. Embrace experimentation in biosecurity governance. Science 368, 138-140 (2020).

11. Palmer, M. J., Fukuyama, F. \& Relman, D. A. A more systematic approach to biological risk. Science 350, 1471-1473 (2015).

\section{Acknowledgements}

M.J.P. receives funding from the Open Philanthropy Project and the Nuclear Threat Initiative. D.A.R. is supported by the Thomas C. and Joan M. Merigan Endowment at Stanford University.

\section{Author contributions}

All authors helped to conceive this work, contributed to the writing of the manuscript and approved the final version of the manuscript.

\section{Competing interests}

The authors declare no competing interests.

\section{Additional information}

Correspondence and requests for materials should be addressed to Jaspreet Pannu or David A. Relman.

Peer review information Nature Protocols thanks Sajal Ghosh and the other, anonymous, reviewer(s) for their contribution to the peer review of this work.

Reprints and permissions information is available at www.nature.com/reprints.

Publisher's note Springer Nature remains neutral with regard to jurisdictional claims in published maps and institutional affiliations.

Received: 16 March 2021; Accepted: 4 November 2021; Published online: 6 December 2021 